\title{
Neumonía por aspiración
}

\section{Aspiration pneumonia}

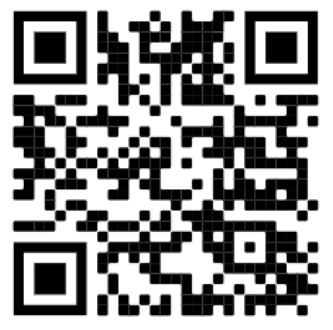

${ }^{1}$ Médico general, graduado de la Universidad de Iberoamérica (UNIBE). Cod MED15963. dr.luisariasv@gmail.com

2 Médica General, graduada de la Universidad de lberoamérica (UNIBE). Cod MED15946.

Danielapelaeze93@gmail.com

${ }^{3}$ Médico general, graduada de la Universidad de lberoamérica (UNIBE). Cod MED16497. glorims03@gmail.com

\author{
${ }^{1}$ Dr. Luis Fernando Arias Villalobos \\ Investigador independiente, Heredia, Costa Rica \\ (iD) https://orcid.org/0000-0001-9072-0068 \\ ${ }^{2}$ Dra. Daniela Peláez Echavarría \\ Investigadora independiente, San José, Costa Rica \\ (iD) https://orcid.org/0000-0003-3519-4282
}

${ }^{3}$ Dra. Gloriana Méndez Solís Investigadora independiente, San José, Costa Rica (iD) https://orcid.org/0000-0003-1306-5025

\begin{abstract}
RECIBIDO
\end{abstract}
CORREGIDO

ACEPTADO

08/09/2020

20/10/2020

03/11/2020

\section{RESUMEN}

La neumonía por aspiración hace referencia a las consecuencias pulmonares ocasionadas por la entrada anormal de líquido, sustancias exógenas en partículas o secreciones endógenas en las vías respiratorias inferiores y, la microaspiración es una característica esencial. Representa casi una sexta parte de los casos de neumonía adquirida en la comunidad que ameritan hospitalización. Los síndromes clínicos con mayor frecuencia y mejor estudiados son neumonitis química, infección bacteriana y obstrucción de las vías respiratorias. La sintomatología abarca un amplio rango que va desde la ausencia de síntomas hasta la insuficiencia respiratoria, además no existe una definición universal, un sistema de calificación de riesgo o un conjunto de criterios diagnósticos específicos. El diagnóstico se basa en una historia clínica con características clínicas de neumonía típica o atípica, apoyada en hallazgos compatibles en la radiografía de tórax u otros estudios de imagen. El tratamiento se basa en medidas de soporte al paciente, aspiración de contenido gástrico y restos en vía aérea, se puede complementar con antibióticos en caso de coincidir con clínica bacteriana, siempre individualizando el origen de la infección ya sea comunitaria o nosocomial y guiados por resultados de muestras microbiológicas. Existen algunas medidas que se han utilizado para ayudar a prevenir la neumonía por aspiración.

PALABRAS CLAVE: neumonía por aspiración; medición de riesgo; características de la residencia; neumonía bacteriana; terapéutica. 


\begin{abstract}
Aspiration pneumonia refers to the pulmonary consequences caused by the abnormal entry of fluid, exogenous substances in particles or endogenous secretions in the lower respiratory tract, and microaspiration is an essential characteristic. It accounts for almost one sixth of the community-acquired pneumonia cases that warrant hospitalization. The most common and beststudied clinical syndromes are chemical pneumonitis, bacterial infection, and airway obstruction. Symptoms encompass a wide range from the absence of symptoms to respiratory failure, and there is no universal definition, a risk rating system or a set of specific diagnostic criteria. The diagnosis is based on a clinical history with clinical characteristics of typical or atypical pneumonia, supported by compatible findings on chest radiography or other imaging studies. Treatment is based on patient support measures, aspiration of gastric content and airway remains, it can be supplemented with antibiotics in case of coinciding with bacterial symptoms, always identifying the origin of the infection, whether community or nosocomial, and guided by results of microbiological samples. There are some measures that have been used to help prevent aspiration pneumonia.
\end{abstract}

KEYWORDS: pneumonia; aspiration; risk assessment; residence characteristics; pneumonia, bacterial; therapeutics.

\title{
INTRODUCCIÓN
}

La neumonía por aspiración representa entre el 5 al $15 \%$ de los casos de hospitalización neumonía adquirida en la comunidad, siendo las poblaciones más afectadas los adultos mayores que provienen de residencias de ancianos 0 instalaciones de cuidados extendidos, quienes representan un $80 \%$ de los casos de esta patología (1-4).

La importancia de la constante actualización radica en que el manejo adecuado y oportuno puede marcar la diferencia sustancial en mortalidad y calidad de vida. La mortalidad de la neumonía por aspiración es superior a la de otras formas de neumonía $(P \leq 0.001)$, y la mortalidad ajustada al riesgo es inferior para los hospitales que informan alta frecuencia de aspiración en comparación con los hospitales que notifican una baja frecuencia de aspiración; esto tiene implicaciones para los hospitales que no hacen una detección oportuna, ya que desde punto de vista administrativo el manejo adecuado puede ser un indicador la calidad del centro hospitalarios (5-7).

El análisis microbiológico de la neumonía por aspiración ha cambiado en los últimos 60 años de infecciones anaeróbicas a ocasionadas bacterias aeróbicas y nosocomiales. Sin embargo, estudios recientes han demostrado que la tendencia en pacientes hospitalizados con sospecha de aspiración se inclina hacia los bacilos gram negativos y cocos gram positivos. Con el creciente aumento de resistencia antimicrobiana se ha propuesto el uso individualizado y racional de antibióticos con el fin de evitar la selección de microorganismos multirresistentes (1-4). La aspiración es normal en sujetos considerados saludables durante el sueño, lo que sugiere que se han desarrollado 
mecanismos de defensa para proteger al sistema de las bacterias aspiradas. Existen hipótesis sobre la etiopatogenia y comprensión de la neumonía por aspiración, la cual se refiere a las consecuencias pulmonares resultantes de la entrada anormal de líquido, sustancias exógenas en partículas o secreciones endógenas en las vías respiratorias inferiores.

La microaspiración es el principal mecanismo patógeno de la mayoría de las neumonías en general, y la microaspiración de contenido orofaríngeo o gastrointestinal alto colonizado condiciona la neumonía por aspiración [4]. De modo que se plantea la necesidad de un enfoque que evalúe estrategias de valoración del riesgo, diagnóstico y tratamiento en pacientes con sospecha de aspiración, especialmente en términos de la capacidad de distinguir los eventos de micro y macro aspiración que conducen a neumonía de aquellos que no.

Por todo lo anterior este artículo pretende realizar una revisión con base a datos y evidencia actualizada disponible sobre aspectos generales de la neumonía por aspiración, así como, estudios recientes sobre el tema y algunos aspectos que aún permanecen controversiales 0 desconocidos.

\section{MÉTODO}

Esta revisión, se realizó mediante consulta de artículos, guías de manejo elaboradas por sociedades como la ATS, IDSA, ACS, de revistas indexadas de validez científica, así como de bases de datos como Up to Date, Pubmed y Chrocrane. Utilizando palabras claves: neumonía por aspiración, valoración del riesgo, adquirida en la comunidad, tratamiento de la neumonía por aspiración. Se compilaron datos de publicaciones con antigüedad no mayor a 5 años, para un total de 19 referencias.

\section{PATOGÉNESIS}

Para que se desarrolle la neumonía por aspiración deben existir un par de condiciones (1):

Compromiso en las defensas habituales que protegen las vías respiratorias inferiores incluyendo, reflejo de la tos, dificultad al tragar o disfagia, deterioro de la conciencia, cierre glótico, entre otros.

Un inóculo deletéreo a las vías respiratorias inferiores, ya sea por un efecto tóxico directo (como el ácido gástrico), el estímulo de un proceso inflamatorio a partir de una infección bacteriana, u obstrucción debido a líquido o partículas sólidas.

Los pulmones están constantemente expuestos a diversas comunidades de microbios de la orofaringe y del aparato gastrointestinal principalmente, y gracias a técnicas independientes cultivo como la reacción en cadena de la polimerasa se ha revelado que los pulmones, antes considerados estériles, pueden estar expuestos a diversas comunidades de microbios. Una hipótesis que vincula el microbioma de las vías respiratorias con neumonía aspiración es que la enfermedad puede producir un cambio en el microbiota pulmonar (disbiosis), que, a su vez, pueden interferir 0 deteriorar las defensas pulmonares $(4,8,9)$. Los cambios fisiopatológicos dependen de la composición del aspirado, las soluciones acidas causan atelectasias, edema alveolar y perdida de surfactante pulmonar. Las partículas causan obstrucción de las vías aéreas pequeñas y necrosis alveolar con posible formación de granulomas alrededor de las partículas de alimentos o antiácidos (10). 


\section{CLASIFICACIÓN DE SÍNDROMES POR ASPIRACIÓN ASOCIADOS}

Los tres síndromes que se ven con más frecuencia clínicamente y mejor estudiados son neumonitis química, infección bacteriana y obstrucción de las vías respiratorias. Aunque ocasionalmente puede haber superposición e incapacidad para clasificar casos individuales. El carácter del inóculo, las manifestaciones, la presentación y las pautas de manejo ayudan a distinguir los síndromes clínicos dentro de la categoría de neumonía por aspiración $(2,10)$.

La neumonía por aspiración es una infección bacteriana del parénquima pulmonar causada por microorganismos específicos, mientras que la neumonitis química es una reacción inflamatoria a los contenidos gástricos irritantes. En casos de aspiración no presenciada, puede ser difícil distinguir entre neumonitis química, neumonía por aspiración y aspiración de materiales sólidos, blandos o partículas; que no son específicamente tóxicas para el pulmón, pero pueden causar obstrucción de las vías respiratorias o el cierre reflejo de las vías respiratorias. Un cuerpo extraño sólido aspirado puede obstruir las vías respiratorias y provocar neumonía postobstructiva, la cual complicaría aún más la distinción de la neumonía bacteriana $(2,4,10)$. Sin embargo, el evento común y desencadenante en todos los casos suele ser la aspiración.

\section{FACTORES PREDISPONENTES PARA ASPIRACIÓN}

En general los pacientes más propensos a aspiración, y macroaspiración se presentan con los siguientes factores de riesgo: alteraciones en la conciencia, por ejemplo, en enfermedades neurológicas como enfermedad cerebro vascular, fármacos o toxinas, anestésicos; alteraciones en el reflejo de tos, como en estados de sedación, coma; dificultad para tragar o disfagia, por alteraciones esofágicas 0 neurológicas; pacientes con mayor riesgo de que el contenido gástrico llegue al pulmón, como en el relujo gastroesofágico $(2,4,7,9,10)$.

Los pacientes pueden presentar combinaciones de pérdida de defensa de la vía área, con reflejos de la vía área alterados, como en estados de coma, anestesia general, intoxicaciones medicamentosas, encefalopatías o enfermedades neuromusculares; alteraciones faríngeas, laríngeas 0 esofágicas (o del esfínter esofágico inferior), presión intrabdominal elevada, como la obesidad, hernia hiatal, embarazo $(2,4,7,9,10)$.

Alteraciones anatómicas como por ejemplo la hernia hiatal se relacionan a menudo con enfermedad por reflujo gastroesofágico (ERGE) sintomática, la cual se considera un factor predisponente para la aspiración. En primer lugar, es importante aclarar lo que significa por ERGE. Reflujo gastroesofágico es el movimiento retrógrado del líquido gástrico en el esófago, y no un estado de exceso gástrico acidez, que es el objetivo de la mayoría de las terapias de ERGE. Además, la ERGE es heterogénea y multifactorial, con múltiples fenotipos identificado en pruebas esofágicas avanzadas y con el apoyo de la actual clasificación de Roma IV esquematiza (por ejemplo, esofagitis erosiva, dispepsia funcional, enfermedad por reflujo no erosiva, y ERGE asintomática) $(11,12)$.

Es posible que la pirosis leve u ocasional no eleve el riesgo de manera significativa. En contraste, los síntomas relacionados con reflujo pasivo, como la regurgitación o gusto acido, pueden indicar un alto riesgo de aspiración pulmonar. Síntomas como tos o 
sibilancias especialmente cuando el paciente estuvo acostado o por las noches pueden indicar un proceso crónico (12).

\section{FACTORES PREDISPONENTES PARA NEUMONÍA POR ASPIRACIÓN Y/O NEUMONITIS QUÍMICA}

No todos los casos de aspiración causaran neumonía, ya que la gravedad del daño pulmonar depende de la composición del aspirado y su volumen. Por lo general en los pacientes en riesgo el $\mathrm{pH}$ gástrico está por debajo de 2.5 y el volumen es mayor a 0.4 $\mathrm{mL} / \mathrm{kg}$ (25 mL). La macroaspiración del contenido gástrico puede conducir a neumonitis química, pero solo con aspiración de gran volumen y $\mathrm{pH}$ por debajo de 2.5 $(2,4,7,9,10)$.

La edad avanzada es un factor de riesgo importante para la neumonía por aspiración, y los pacientes institucionalizados y aquellos con comorbilidades son particularmente vulnerables $(2,3)$.

Se estima al menos $10 \%$ de los pacientes con neumonía adquirida en la comunidad sufren aspiración en comparación con el $30 \%$ de los que tenían neumonía asociada a cuidados de la salud en pacientes adultos mayores institucionalizados.

Además, Suele existir una asociación con la enfermedad periodontal, y la infección bacteriana es menos común en pacientes con buena higiene dental o dentición (2-4). De igual manera que en las neumonías de otros tipos, las comorbilidades del paciente conllevan un peor desenlace clínico, en especial las pulmonares como el EPOC $(1,3,7)$.

\section{CARACTERÍSTICAS CLÍNICAS}

Las características clínicas van desde la ausencia de síntomas hasta la dificultad grave con insuficiencia respiratoria. La aspiración puede afectar las vías respiratorias (causando broncoespasmo, asma y tos crónica) o el parénquima pulmonar (neumonía, neumonitis química) (4). El curso clínico se puede dar de manera súbita (en el caso de neumonitis o afectación a las vías respiratorias), aguda y subaguda, o lenta y progresivamente. Los hallazgos de presentación en la neumonía por aspiración debida a una infección bacteriana son muy variables, con síntomas que se desarrollan en cuestión de horas a unos días después de un evento de aspiración, según el momento en que se valore al paciente durante el curso de la infección, las bacterias involucradas y el estado inmunológico del huésped $(2,4,7)$. La epidemiología de la neumonía sugiere una fuerte correlación con el aumento de la edad, por lo que los ancianos son particularmente susceptibles a eventos adversos secundarios como sepsis, Síndrome de dificultad respiratoria aguda (SDRA) y muerte $(2-4,7,12)$.

La mayoría de los pacientes presentan manifestaciones comunes de neumonía, como tos, fiebre, esputo purulento y disnea. Los casos que implican anaerobios suelen evolucionar durante varios días o semanas en lugar de horas, en parte por bacterias menos virulentas, y los escalofríos son poco frecuentes, sin embargo, que muchos pacientes presentan posteriormente complicaciones caracterizadas por supuración y necrosis, incluyendo absceso pulmonar, neumonía necrotizante o empiema. Con la aspiración ácida o las infecciones que implican S. aureus o bacilos gramnegativos, la evolución es mucho más rápida (2). En imágenes el segmento pulmonar involucrado depende de la posición del paciente durante el evento de aspiración. Si el evento de aspiración ocurre mientras el paciente está en decúbito, el 
desarrollo de neumonía es más común en los segmentos posteriores de los lóbulos superiores y los segmentos apicales de los lóbulos inferiores; mientras que si ocurre mientras el paciente está en posición vertical, la ubicación cambia a los segmentos basales de los lóbulos inferiores y es posible que la radiografía de tórax no muestre cambios significativos como infiltrados bilaterales hasta varias horas posterior a la aspiración (2-4,7,9,10).Por otra parte, la neumonitis química se caracteriza por una aparición repentina de disnea, hipoxemia, taquicardia y sibilancias difusas 0 crepitaciones, cianosis, fiebre de bajo grado. El curso de esta enfermedad varía, y hasta un $15 \%$ de los pacientes pude presentar SRDA en casos de aspiración presenciada. Más de la mitad de los pacientes presenta una rápida mejoría clínica la cual se refleja en la radiografía de tórax, sin embargo, se ha visto que hasta un cuarto de los enfermos tiene mejora rápida inicial, pero luego desarrolla nuevos infiltrados en la radiografía de tórax, lo que podría indicar una infección bacteriana secundaria o el desarrollo de SDRA superpuesto al pulmón lesionado por ácido $(2,4,10)$.

Es posible el edema pulmonar por aumento de la permeabilidad ante la lesión pulmonar, hipertensión pulmonar e hipercapnia con acidosis respiratoria. La hipotensión sugiere inundación alveolar y se relaciona con lesión pulmonar masiva. Muchos de estos pacientes tienen resolución de los síntomas dentro de las 24 a 48 horas y solo requieren apoyo tratamiento, sin antibióticos. Lo cual es indicativo de neumonitis química (1).

\section{DIAGNÓSTICO}

El diagnóstico de la neumonía por aspiración es difícil, en parte porque no existe una definición universal o un conjunto de criterios de diagnóstico. Depende de una historia clínica característica (macroaspiración presenciada) y factores de riesgo, debe haber evidencia de signos y síntomas clínicos de neumonía (típicos o atípicos), y hallazgos compatibles en la radiografía de tórax u otros estudios de imagen. Se suelen tomar muestras para tinción de Gram, cultivos y PSA (prueba de sensibilidad antibiótica) en la medida de lo posible, además permite guiar la antibioticoterapia $(3,4)$.

Las muestras protegidas con cepillo y lavado broncoalveolar protegido para cultivo tienen mejores resultados, no obstante, los resultados nunca están listos para la toma de decisiones con respecto a la antibioticoterapia inicial, la cual, en si misma puede llegar influir en los resultados de los cultivos. Por otra parte, los microrganismos anaerobios los cuales han atribuido típicamente a la neumonía por aspiración muchas veces son difícil de cultivar y toman más tiempo en comparación con bacterias aerobias. No se recomienda la recolección de muestras para tinción de Gram o cultivos en pacientes ambulatorios (1-4,7).

En el escenario apropiado, el diagnóstico de neumonía por aspiración se apoya con una imagen representativa de neumonía. En general, la sensibilidad de una radiografía de tórax para diagnosticar neumonía varía entre $32 \%$ y $77,7 \%$ (3). Las tomografías computarizadas (TC) de tórax sin contraste siguen siendo estándar de referencia para el diagnóstico de neumonía y actualmente se inclina a tener el mayor impacto en el diagnóstico y las decisiones de tratamiento posteriores. Por lo que, si los riesgos de exposición a la radiación no son una preocupación para el paciente, se recomienda utilizar imágenes de TC sin contraste siempre que el diagnóstico esté en duda hasta que los estudios futuros aclaren 
el enfoque más apropiado para las imágenes $(3,13)$.

\section{VALORACIÓN DE LA SEVERIDAD}

Las características únicas y la gravedad de la enfermedad en la presentación clínica de la neumonía por aspiración han hecho que los sistemas de puntuación tradicionales como CURB-65 o Pneumonia Severity Index (PSI) no puedan pronosticar con precisión la gravedad de los pacientes que desarrollan neumonía por aspiración en la presentación inicial. Dado que la neumonía por aspiración parece ser más común en pacientes que tienen múltiples comorbilidades, CURB-65 ha sido poco confiable en esta población $(1,7)$. La Sociedad Americana del Tórax (ATS) y la Sociedad Americana de Enfermedades infecciosas (IDSA) en sus guías y pautas para neumonía adquirida en la comunidad (NAC) del 2007 recomendaban un conjunto de dos criterios mayores y nueve menores para ayudar a diagnosticar NAC grave y pacientes que requerirán ingreso a cuidados intensivos. Varios grupos han validado estos criterios en cohortes de neumonía en diferentes países. En sus más recientes guías para NAC, publicadas en 2019, siguen recomendando los criterios para NAC severo de IDSA / ATS del 2007 sobre otros puntajes publicados, porque están compuestos de parámetros de severidad fácilmente disponibles y son más precisos que los otros puntajes descritos anteriormente (1).

Sin embargo, estos criterios tampoco especifican la neumonía por aspiración como una entidad única de la NAC. En general, hay una falta de un sistema de calificación de riesgo que se centre específicamente en la neumonía por aspiración para predecir la gravedad o la mortalidad $(1,7)$
Se ha desarrollado recientemente una Calculadora de Riesgo Quirúrgico (SRC) genérica, por el Colegio americano de Cirujanos, la cual compara 21 variables preoperatorias de pacientes con una gran base de datos de resultados de pacientes del Programa Nacional de Mejoramiento de la Calidad Quirúrgica de los Estados Unidos, para ayudar a predecir el riesgo de complicaciones postoperatorias, y su validación ha mostrado una buena precisión en la predicción de neumonía (14).

En un reciente estudio observacional prospectivo multicentro se Identificaron cinco variables asociadas de forma independiente con la neumonía posoperatoria: estado funcional [odds ratio (OR) 2,28; intervalo de confianza (IC) del 95\%: 1,58 a 3,12]; valores de $\mathrm{SpO} 2$ preoperatorios mientras se respira aire ambiental (OR 0,83; IC del 95\%: 0,78 a 0,84); administración de soluciones coloides intraoperatorio (OR 2,97; IC del 95\%: 1,94 a $3,99)$; transfusión de sangre intraoperatoria (OR 2,19; IC del 95\%: 1,41 a 4,71); y sitio quirúrgico (cirugía abdominal superior abierta OR 3,98; IC del 95\% 2,19 a 7,59). Sin embargo, el modelo debe ser validado externamente, para evaluar su desempeño en otras áreas geográficas y con un grupo quirúrgico diferente. Con una cohorte quirúrgica de 5094 pacientes, se produjo neumonía posoperatoria en un $2,4 \%$ de los pacientes (120). Los pacientes con neumonía que requirieron ingreso en la $\mathrm{UCl}$ fueron 82 de los 120 (68,3\%), en comparación con 399 de los 4974 (8,0\%) sin neumonía $(P<0,001)(15)$.

\section{MICROBIOLOGÍA}

Estudios que evaluaron la microbiología de pacientes con neumonía por aspiración en la década de 1970 mostraron altas tasas de 
aislamiento de organismos anaeróbicos; Sin embargo, estos estudios a menudo utilizaron muestras transtraqueales y evaluaron a los pacientes al final del curso de la enfermedad, factores que pudieron haber contribuido a una mayor probabilidad de identificar organismos anaerobios. Principalmente se aislaron F. nucleatum, Bacteroides spp distintas de $B$. fragilis y Prevotella. Por el contrario, evidencia más reciente ha sugerido que las bacterias anaerobias no juegan un papel tan importante en la etiología (1-4).

Una reciente revisión sistemática que incluyo 8 estudios los cuales utilizaron técnicas de muestreo diferentes, y para identificar microorganismos. Las técnicas invasivas, como la broncoscopia, recuperaron más bacterias anaeróbicas que las técnicas menos invasivas, como esputo y hemocultivos que aislaron principalmente bacterias aeróbicas. Los organismos Gram negativos más comunes fueron Escherichia coli, K. pneumoniae y $P$. aeruginosa, y los organismos Gram positivos más comunes fueron $S$. aureus y $S$. pneumoniae. Muchos estudios reportaron un crecimiento mixto. Los resultados están limitados por la escasa sensibilidad y especificidad de los aislamientos respiratorios y la administración previa de antibióticos en algunos estudios (16).

La neumonía por aspiración adquirida en el hospital y en adultos mayores institucionalizados suele incluir a S. aureus o bacilos gramnegativos, como $P$. aeruginosa, que pueden colonizar la cavidad bucal o catéteres de los pacientes hospitalizados (1$4,7,9,10)$.

\section{TRATAMIENTO}

El tratamiento inicial de la aspiración gástrica requiere manejo y mantenimiento de las vías respiratorias, tratamiento del edema 0 broncoespasmo y la minimización del daño tisular. Según la demanda de cuidados requerida y la gravedad de la neumonitis, el tratamiento puede incluir aspiración, broncoscopia, intubación, ventilación mecánica y cuidados intensivos $(2,4)$.

En cuanto se sospeche de regurgitación debe colocarse al paciente en posición con la cabeza hacia abajo para que el contenido gástrico drene por la boca y no hacia la tráquea, se realiza aspiración de la faringe y tráquea a ser posible. La mayoría de veces están indicados la broncoscopia con lavado bronquial cuando hubo aspiración de partículas.

La base del tratamiento en pacientes que desarrollan hipoxemia es la ventilación con presión positiva, a menudo se requieren intubación y ventilación con presión positiva al final de la espiración (PEEP) o presión positiva continua en la vía aérea (CPAP). Casi nunca se recomienda el uso de corticoesteroides. $(1,2,4,7)$.

Para los pacientes con sospecha de neumonía por aspiración, la decisión sobre la terapia con antibióticos depende del lugar de adquisición: comunidad, hospital o centro de atención de pacientes institucionalizados. Se administran antibióticos a pacientes que tienen un episodio de aspiración y una radiografía de tórax anormal, aunque incluso con una radiografía inicialmente normal, se administran antibióticos a aquellos con enfermedad grave (es decir, enfermedad caracterizada por shock o que requiere intubación).

$\mathrm{Si}$ se sospecha neumonitis química, inicialmente no se recomiendan antibióticos, incluso con una radiografía anormal, a menos que el paciente esté gravemente enfermo; en pacientes con enfermedad leve a moderada y una radiografía anormal, la recomendación es suspender los 
antibióticos y reevaluar al paciente en 48 horas $(1-4,7,9,10)$. Los pacientes con comorbilidades enfermedades graves se tratan sobre la base de los riesgos asociados, con su salud dental y la multi drogo resistencia (MDR). El tratamiento de rutina para los patógenos anaeróbicos no es necesario en pacientes con salud dental normal, pero debe considerarse en aquellos con mala salud dental, neumonía necrosante o absceso pulmonar (2-4,7).

Si el sitio de adquisición es la comunidad, el tratamiento ambulatorio de primera linea es con amoxicilina-clavulanato (875 mg por vía oral dos veces al día), también se puede usar moxifloxacino (400 mg por vía oral o IV una vez al día), levofloxacina (750 mg una vez al día oral o IV) o clindamicina para los pacientes que tienen una alergia grave a la penicilina (450 mg por vía oral tres veces al día), si existe sospecha de anaerobios y se requiere terapia parenteral, se recomienda ampicilina-sulbactam (1,5 a $3 \mathrm{~g}$ IV cada 6 horas) como terapia de primera línea $(2,4)$.

Para la neumonía por aspiración adquirida en el hospital 0 centros de pacientes institucionalizados, la mayoría de las autoridades consideran que las bacterias aerobias, especialmente los bacilos gramnegativos y $S$. aureus, son más importantes que los anaerobios; por lo general se detectan fácilmente con un crecimiento abundante a partir de muestras adecuadas, por lo que la terapia debe estar dirigido a los organismos aerobios. Antes de que los resultados del cultivo y la sensibilidad antibiótica estén disponibles, si hay sospecha de colonización con $\mathrm{S}$. aureus meticilino resistente (MRSA) se puede considerar la adición de vancomicina (15 mg / kg cada 12 h, IV) o linezolid (600 mg cada $12 \mathrm{~h}$, oral o IV) los cuales se deberían suspender sino se detecta el MRSA (1$4,7,9,10)$.
En estos casos, cuando se percibe la necesidad de tratar bacterias anaerobias, ( $p$. Ej., pacientes con mala dentición e higiene) generalmente se recomienda un régimen con actividad tanto contra aerobios como anaerobios; así mismo como bacilos gramnegativos aerobios resistentes (p. Ej., en pacientes que se sabe que están colonizados con bacilos gramnegativos resistentes o pacientes que han recibido antibióticos por vía intravenosa en los últimos 90 días), recomiendan antibióticos de amplio espectro como los carbapenémicos como imipenem $(500 \mathrm{mg}$ cada 6 horas $01 \mathrm{~g}$ cada 8 horas IV) 0 meropenem (1 g cada $8 \mathrm{~h}, \mathrm{IV}$ ) o piperacilinatazobactam (4,5 g cada 8 horas IV), ya que estos agentes cubrirán prácticamente todos los anaerobios, así como la mayoría de los bacilos gramnegativos aerobios. Todos los regímenes sugeridos aumentan el riesgo de infección por C. difficile, (frecuentemente asociadas con el uso de clindamicina) especialmente en pacientes con otros factores de riesgo como hospitalización, edad avanzada y enfermedad grave (1-4,7). La duración de los antibióticos es arbitraria y no está bien estudiada. La duración habitual de la terapia para los casos que no se complican por cavitación o empiema es de 7 días Los pacientes con derrames pleurales asociados deben tener una toracocentesis para excluir el empiema, que a menudo es complicación de neumonía por anaerobios. Los pacientes con absceso pulmonar necesitan un curso más largo de antibióticos, por lo general hasta que hay aclaramiento radiográfico o mejora significativa, como una pequeña lesión residual estable (2).

La revisión sistemática mencionada en el apartado de microbiología destaca la escasez de datos que orienten el tratamiento de la neumonía por aspiración. Los diferentes regímenes incluyen penicilina $G$ 
como terapia inicial, ampicilina / sulbactam, clindamicina +/- cefalosporina, azitromicina y carbapenémicos. Descubrieron que, en general, en todos los estudios, la elección de cobertura fue en su mayor parte de amplio espectro para cubrir tanto bacterias Gram positivas como Gram negativas, de modo que se respalda el uso de antibióticos de amplio espectro, pero debe tenerse en cuenta el problema de la resistencia generada, esto abonado al lento desarrollo de nuevos agentes (16).

\section{PREVENCIÓN}

La literatura internacional no revela consenso claro sobre cuáles y cuántas características y factores de riesgo deben estar presentes para diagnosticar y distinguir la neumonía (adquirida en centros de pacientes institucionalizados para adultos mayores) y la neumonía por aspiración. Para considerar la neumonía por aspiración en los residentes de hogares de ancianos, el panel de médicos llego a un consenso final sobre los indicadores de riesgo: disfagia, incidente de asfixia atragantamiento, (antecedentes de) alimentación por sonda, enfermedad neurológica y deterioro cognitivo (17).

En una cohorte retrospectiva se vio que la profilaxis para pacientes con neumonitis por aspiración aguda con antibióticos no ofrece un beneficio clínico y puede generar selección de baterías resistentes que resultan en la necesidad de intensificar la terapia con antibióticos entre aquellos que si desarrollan neumonía por aspiración (18). Un metaanálisis que incluyo 26 estudios demostró de manera significativa que el uso de inhibidores de bomba de protones (IBP) en pacientes ambulatorios se asocia con un riesgo 1,5 veces mayor de NAC, con el mayor riesgo dentro de los primeros 30 días después del inicio de la terapia, independientemente de la dosis de IBP o la edad del paciente. La terapia con IBP también aumentó el riesgo de hospitalización por NAC. Por lo tanto, la aspiración puede ser un objetivo de intervenciones clínicamente efectivas, pero una mala comprensión del mecanismo de aspiración puede conducir a resultados adversos (19).

Varias medidas pueden ayudar a prevenir la neumonía por aspiración sin introducir morbilidad, incluyen intervenciones dietéticas para la disfasia, cuidado bucal, alimentación por sonda pospilórica y la posición semisentada para pacientes en ventilación mecánica (10).

\section{CONCLUSIONES}

A pesar de la enseñanza original, la neumonía por aspiración es difícil de distinguir de otros síndromes de neumonía. La microbiología de la neumonía después de una macroaspiración ha cambiado en los últimos 60 años de una infección anaeróbica a una de bacterias aeróbicas y nosocomiales.

Se necesitan ensayos clínicos que evalúen las estrategias de diagnóstico y tratamiento en pacientes con sospecha de aspiración, especialmente en términos de la capacidad de distinguir los eventos de microaspiración y macroaspiración que conducen a una infección del tracto respiratorio inferior con respecto de aquellos que no resultan en una infección. No existen criterios o sistema de calificación de riesgo que delimiten la neumonía por aspiración como una entidad independiente de neumonía adquirida en la comunidad 0 en centros de pacientes institucionalizados para predecir la gravedad o la mortalidad.

Se ha visto que los casos de neumonía que involucran aspiración suelen tener mayor 
riesgo de complicaciones y peor desenlace clínico por lo que es crucial seguir investigando en este campo. Existen medidas que podrían ayudar a prevenir la neumonía por aspiración sin introducir morbilidad, sin embargo, algunas han sido motivo de controversia.

\section{REFERENCIAS}

1. Metlay JP, Waterer GW, Long AC, Anzueto A, Brozek J, Crothers K, et al. Diagnosis and Treatment of Adults with Community-acquired Pneumonia. An Official Clinical Practice Guideline of the American Thoracic Society and Infectious Diseases Society of America. Am J Respir Crit Care Med [Internet]. 2019 Oct 1 [citado 20 de agosto de 2020];200(7):e45-67.https://doi.org/10.1164/rccm.201908-1581ST

2. Bartlett J. G. Aspiration pneumonia in adults [Internet]. Uptodate. 2020. [citado 20 de agosto de 2020]. Disponible:https://www.uptodate.com/contents/aspiration pneumonia-in adults?search=Aspiration\%20pneumonia\&source=search result\&selectedTitle=1 150\&usage type=default\&dis play rank=1

3. Makhnevich A. Aspiration Pneumonia in Older Adults. J Hosp Med [Internet]. 2019 [citado 20 de agosto de 202014(7):429. https://doi.org/10.12788/jhm.3154

4. Mandell LA, Niederman MS. Aspiration Pneumonia. New England Journal of Medicine [Internet]. 14 de febrero de 2019 [citado 24 de agosto de 2020];380(7):651-63.https://doi.org/10.1056/NEJMra1714562

5. Lindenauer PK, Strait KM, Grady JN, Ngo CK, Parisi ML, Metersky M, et al. Variation in the Diagnosis of Aspiration Pneumonia and Association with Hospital Pneumonia Outcomes. Annals ATS [Internet]. 3 de enero de 2018 [citado 24 de agosto de 2020];15(5):562-9.https://doi.org/10.1513/AnnalsATS.201709-7280C

6. Komiya K, Rubin BK, Kadota J, Mukae H, Akaba T, Moro H, et al. Prognostic implications of aspiration pneumonia in patients with community acquired pneumonia: A systematic review with meta-analysis. Scientific Reports [Internet]. 7 de diciembre de 2016 [citado 3 de septiembre de 2020];6(1):1-9. https://doi.org/10.1038/srep38097

7. Rodriguez AE, Restrepo MI. New perspectives in aspiration community acquired Pneumonia. Expert Review of Clinical Pharmacology [Internet]. 2019 Oct 3 citado 3 de septiembre de 2020];12(10):991-1002. https://doi.org/10.1080/17512433.2019.1663730

8. Dickson RP, Erb-Downward JR, Martinez FJ, Huffnagle GB. The Microbiome and the Respiratory Tract. Annu Rev Physiol [Internet]. 10 de febrero de 2016 [citado 4 de septiembre de 2020];78(1):481-504. https://doi.org/10.1146/annurev-physiol-021115-105238

9. Neill S, Dean N. Aspiration pneumonia and pneumonitis: a spectrum of infectious/noninfectious diseases affecting the lung. Current Opinion in Infectious Diseases [Internet]. abril de 2019 [citado 4 de septiembre de 2020];32(2):152-157. https://doi.org/10.1097/QCO.0000000000000524

10. DiBardino DM, Wunderink RG. Aspiration pneumonia: A review of modern trends. Journal of Critical Care [Internet]. 2015 Feb 1 [citado 4 de septiembre de 2020];30(1):40-8. https://doi.org/10.1016/.j.jcrc.2014.07.011 
11. Drossman DA. Functional Gastrointestinal Disorders: History, Pathophysiology, Clinical Features, and Rome IV. Gastroenterology [Internet]. 2016 May 1 [citado 5 de septiembre de 2020];150(6):1262-1279.e2. https://doi.org/10.1053/i.gastro.2016.02.032

12. Lee AS, Ryu JH. Aspiration Pneumonia and Related Syndromes. Mayo Clinic Proceedings [Internet]. 2018 Jun 1 [citado 5 de septiembre de 2020];93(6):752-62. https://doi.org/10.1016/j.mayocp.2018.03.011

13. Claessens Y-E, Debray M-P, Tubach F, Brun A-L, Rammaert B, Hausfater P, et al. Early Chest Computed Tomography Scan to Assist Diagnosis and Guide Treatment Decision for Suspected Community-acquired Pneumonia. Am J Respir Crit Care Med [Internet]. 13 de julio de 2015 [citado 5 de septiembre de 2020];192(8):974-82. https://doi.org/10.1164/rccm.201501-00170C

14. Ajitsaria P, Eissa SZ, Kerridge RK. Risk Assessment. Curr Anesthesiol Rep [Internet]. 2018 [citado 5 de septiembre de 2020];8(1):1-8. https://doi.org/10.1007/s40140-018-0246-9

15. Russotto V, Sabaté S, Canet J, Network for the P groupof the ES of A (ESA) CT. Development of a prediction model for postoperative pneumonia: A multicentre prospective observational study. European Journal of Anaesthesiology | EJA [Internet]. febrero de 2019 [citado 5 de septiembre de 2020];36(2):93-104. https://doi.org/10.1097/EJA.0000000000000921

16. Bowerman TJ, Zhang J, Waite LM. Antibacterial treatment of aspiration pneumonia in older people: a systematic review. Clin Interv Aging [Internet]. 30 de octubre de 2018 [citado 7 de septiembre de 2020];13:2201-13. https://doi.org/10.2147/ClA.S183344

17. Hollaar V, van der Maarel-Wierink C, van der Putten G-J, van der Sanden W, de Swart B, de Baat C. Defining characteristics and risk indicators for diagnosing nursing home-acquired pneumonia and aspiration pneumonia in nursing home residents, using the electronically-modified Delphi Method. BMC Geriatrics [Internet]. 7 de marzo de 2016 [citado 7 de septiembre de 2020];16(1):60. https://doi.org/10.1186/s12877-016-0231-4

18. Dragan V, Wei Y, Elligsen M, Kiss A, Walker SAN, Leis JA. Prophylactic Antimicrobial Therapy for Acute Aspiration Pneumonitis. Clin Infect Dis [Internet]. 1 de agosto de 2018 [citado 8 de septiembre de 2020];67(4):513-8. https://doi.org/10.1093/cid/ciy 120

19. Lambert AA, Lam JO, Paik JJ, Ugarte-Gil C, Drummond MB, Crowell TA. Risk of Community-Acquired Pneumonia with Outpatient Proton-Pump Inhibitor Therapy: A Systematic Review and Meta-Analysis. PLoS One [Internet]. 2015 Jun 4 [citado 8 de septiembre de 2020];10(6). https://doi.org/10.1371/journal.pone.0128004 